Article

\title{
High-Voltage Drivers Based on Forming Lines with Extended Quasi-Rectangular Pulses for High-Power Microwave Oscillators
}

\author{
Vladislav V. Rostov *(1), Alexei S. Stepchenko, Pavel V. Vykhodtsev and Ruslan V. Tsygankov
}

Citation: Rostov, V.V.; Stepchenko, A.S.; Vykhodtsev, P.V.; Tsygankov, R.V. High-Voltage Drivers Based on Forming Lines with Extended Quasi-Rectangular Pulses for High-Power Microwave Oscillators. Electronics 2022, 11, 406. https:// doi.org/10.3390/electronics11030406 Academic Editor: Leonardo Pantoli

Received: 14 December 2021

Accepted: 24 January 2022

Published: 28 January 2022

Publisher's Note: MDPI stays neutral with regard to jurisdictional claims in published maps and institutional affiliations.

Copyright: (C) 2022 by the authors. Licensee MDPI, Basel, Switzerland. This article is an open access article distributed under the terms and conditions of the Creative Commons Attribution (CC BY) license (https:// creativecommons.org/licenses/by/ $4.0 /)$.
Institute of High Current Electronics, Siberian Branch of Russian Academy of Science, Tomsk 634055, Russia; stepchenko@lfe.hcei.tsc.ru (A.S.S.); pasha@lfe.hcei.tsc.ru (P.V.V.); tsygankov@lfe.hcei.tsc.ru (R.V.T.)

* Correspondence: rostov@lfe.hcei.tsc.ru; Tel.: +7-3812-491641

\begin{abstract}
The paper considers such modifications of an ordinary pulse-forming line (PFL) as doublewidth and triple-width forming lines (DWFL, TWFL) built around the PFL by nesting one and two additional uncharged lines, respectively, into its free volume inside the inner conductor of the PFL. The theoretical analysis is supported by simulation and experimental data, showing that the TWFL provides a 3-fold increase in the voltage pulse width and that it can be further increased by an arbitrary integer factor $k$. The results of the numerical simulations also show the electric field behavior and other features, including the edge effect in the TWFL. The proposed method opens up new opportunities for designing compact high-power microwace (HPM) sources.
\end{abstract}

Keywords: pulsed power; pulse-forming line; explosive emission; high-current electron beam; Cherenkov-type oscillator

\section{Introduction}

High-voltage drivers based on distributed pulse-forming lines (PFLs) [1] are an excellent power supply for high-power microwave (HPM) oscillators. This type of driver can provide relatively short rise times (of a few nanoseconds) and rectangular voltage pulses that are, in all respects, more beneficial than those formed by Marx generators or modular pulse-forming networks (PFNs) [2]. The only worthy exception is an innovative "zigzag" PFN Marx design that provides a pulse rise time of $5 \mathrm{~ns}$ at an output voltage of $400 \mathrm{kV}$ in a beam diode of $100 \Omega$ (burst mode operation for $10 \mathrm{~s}$ at $100 \mathrm{~Hz}$ ) [3].

Shortening the voltage rise is exceptionally important for stable cathode operation during explosive electron emission [4-7]. It is likely that this weakens the space charge effect at emission centers [8]. If the rise time is also sharpened to $\approx 10^{-9} \mathrm{~s}$ or less [4,9], one can attain a picosecond EEE accuracy [10] and phase synchronization of parallel microwave channels [11,12]. In addition, shortening the voltage rise decreases the microwave oscillator transit time [11] and increases the efficiency of the beam-to-wave energy conversion [13,14]. Finally, constant beam parameters within the pulse plateau are needed for mode selection, especially in oversized slow wave structures, where one mode should dominate over competitors for as long as possible $[15,16]$. The advantages of drivers with distributed PFLs were proved, even by the first experiments on millimeter-wavelength relativistic oscillators $[17,18]$ and, later, on $\mathrm{Ka}, \mathrm{X}$, and S-band repetitively pulsed backward wave oscillators [5,12-14,19-22].

For the practical application of gigawatt and sub-gigawatt, repetitively pulsed electromagnetic sources with pulses longer than 10-20 ns, a decrease in their dimensions is needed. This is one of the problems that we consider in this paper. The basic features of pulse-forming lines and innovative ways of dimension reduction are analyzed. Our attention was focused on pulse extension, for which we further developed the fruitful idea of pulse width doubling [23] by using an additional, uncharged inner line. This approach 
has shown obvious advantages in experiments with a double-width PFL [14]. To prove the concept, the laboratory model of a triple-width PFL was created and tested.

\section{Basic Properties of a Single FL}

The outer dimensions of a single PFL, as a coaxial segment (Figure 1), are determined by its length $L_{\mathrm{FL}}$ and the diameter of its outer conductor $2 r_{2}$.

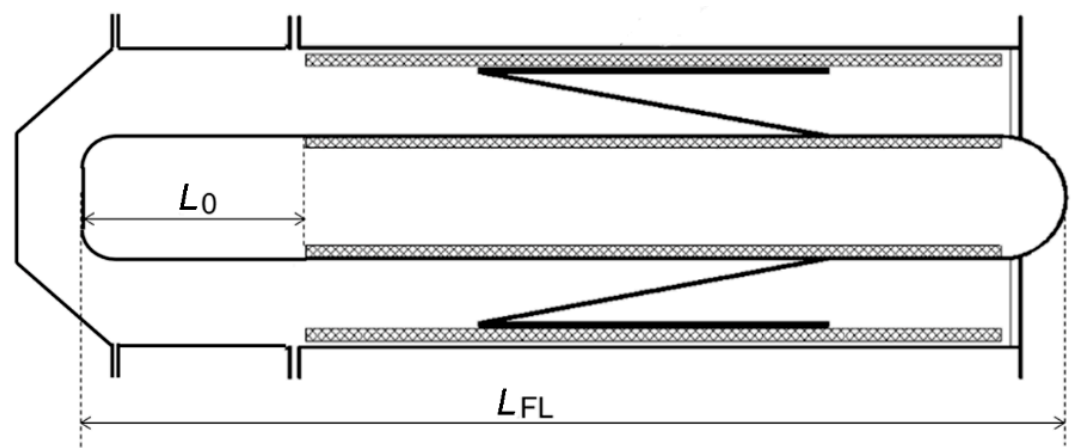

Figure 1. Geometry of PFL with built in Tesla transformer. The main part of the length $L_{\mathrm{FL}}$ is a Tesla transformer range (hatched area shows the magnetic cores). An additional piece of coaxial line $L_{0}$ can be used to increase the formed voltage pulse width.

The wave impedance of the PFL and the electric strength at its inner conductor (at diameter $2 r_{1}$ ) can be written as:

$$
\begin{gathered}
\rho=60 \Lambda / \varepsilon^{1 / 2} \Omega, \text { where } \Lambda=\ln \left(r_{2} / r_{1}\right) ; \text { and } \\
E=U_{0} /\left(r_{1} \Lambda\right)
\end{gathered}
$$

where $\varepsilon$ is the relative dielectric permittivity of liquid insulation and $U_{0}$ is the PFL charging voltage before high-voltage switch operation. The high-voltage switch (not shown in Figure 1 to the right) is normally a spark-gap switch operated with $N_{2}$ at a pressure of $\approx 1 \mathrm{MPa}$. Neglecting the edge effects, the PFL stored energy $W$ and the wave propagation time $T$ are expressed as:

$$
W=T U_{0}{ }^{2} / 2 \rho \text { with } T=L_{\mathrm{FL}} \varepsilon^{1 / 2} / \mathcal{c}
$$

where $c$ is a light speed in free space. As it is well known, after the discharge process, the formed at the load voltage pulse width is $\tau_{p}=2 T$. With the field strength and PFL outer dimension kept constant, varying $r_{1}$ (or $\Lambda$ ) gives the condition of maximum stored energy $\Lambda^{*}=0.5$ and $\rho^{*}=30 / \varepsilon^{1 / 2}$. Thus, for insulation with ordinary transformer oil $(\varepsilon=2.25)$, we have $\rho^{*}=20 \Omega[1]$.

Typically, PFLs are charged with a built-in Tesla transformer, which was originally proposed by [24] and applied to repetitively-pulsed, high-current SINUS accelerators [1,25,26]. In the SINUS accelerators, the transformer comprises cylindrical magnetic cores, a primary winding with one turn, and a secondary winding of approximately 1500 turns. The magnetic coupling ratio between the primary and secondary windings is close to unity if the length of the magnetic cores is many times larger than the radial gap width and the length of the coils is no greater than half the core length. Another feature of the SINUS accelerators (including version SINUS-4 [24]) is the use of thyristors in the primary transformer circuit for energy switching from capacitors charged to $\approx 500 \mathrm{~V}$, which allows one to power an accelerator from commercial supply lines without additional step-up transformers. The use of such semiconductor switches in the primary transformer circuit determines the charging time $t_{\mathrm{ch}}$ for PFLs as a coaxial capacitor: from a few microseconds at a voltage pulse width of $4 \mathrm{~ns}$ in compact accelerators (SINUS-120, $r_{2}=6 \mathrm{~cm}, L_{\mathrm{FL}}=40 \mathrm{~cm}$ [20]) to several tens of microseconds in large setups (SINUS-7, $r_{2}=50 \mathrm{~cm}, L_{\mathrm{FL}}=500 \mathrm{~cm}$ ) [1]. With a spiral line instead of $L_{0}$ (Figure 1), the voltage pulse can be lengthened up to 130 ns $[1,13]$. 
Before proceeding to more complex equivalent circuits, let us consider the discharge circuit of a single PFL with an electrical length $T$ and load resistance $R_{\mathrm{L}}$ (Figure 2).

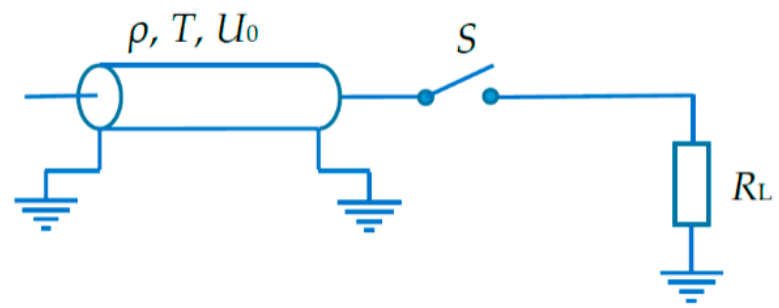

Figure 2. Equivalent discharge circuit of single coaxial PFL.

Upon operation of its switch $(S)$, a current $I=U_{0} /\left(R_{\mathrm{L}}+\rho\right)$ arises in the circuit and a voltage pulse $U_{\mathrm{L}}=U_{0} R_{\mathrm{L}} /\left(R_{\mathrm{L}}+\rho\right)$ at the load. In the same time, a voltage wave of amplitude $U_{\mathrm{b}}=U_{0} \rho /\left(R_{\mathrm{L}}+\rho\right)$ propagates in the line. At $t=T$, the wave front reaches the open end of the line and goes back with the same sign. At $t=2 T$, the formation of the first (main) pulse is completed. When $R_{\mathrm{L}}=\rho$, there is no reflection from the load, and the energy stored in the PFL is fully transmitted to the load as a main pulse of duration $2 T$ and amplitude equal to half the charge voltage $U_{0}$.

It should be noted that the voltage pulse width at the load $\tau_{p}=2 T$ is about three orders of magnitude shorter than the PFL charging time $t_{\mathrm{ch}}$. Thus, for pulses of $10 \mathrm{~ns}$ and shorter, the PFL length is $L_{\mathrm{FL}} \leq 100 \mathrm{~cm}$ [26-29], and for pulses of $100 \mathrm{~ns}$, it should be 10 times longer. The problem of decreasing the PFL length can be solved by using high-permittivity liquids (treated water, alcohol, glycerol, etc.), but this method involves two problems. First, it greatly increases the conductivity [30,31], and this necessitates the use of extremely short PFL charging, hence the abandonment of thyristors in the primary circuit [1]. Second, at $U_{\mathrm{L}} \leq 400 \mathrm{kV}$, the optimum diode impedance of Cherenkov-type relativistic HPM oscillators is $R_{\mathrm{L}} \approx 100 \Omega$ or higher, and cannot be decreased without a loss in their efficiency $[13,32,33]$. At arbitrary PFL and load impedances, there is an energy loss: a series of reflected pulses in addition to the main one at the load. The efficiency of energy transfer from the PFL to the first pulse is given by:

$$
\eta=\left(4 R_{\mathrm{L}} \cdot \rho\right) /\left(R_{\mathrm{L}}+\rho\right)^{2}
$$

As can be seen, the efficiency drops when the permittivity is high and $\rho<<R_{\mathrm{L}}$. This does not exclude the use of modern high-permittivity dielectric liquids, but suggests the necessity of special measures for matching the PFL and the load. For example, such a liquid can be MIDEL 7131, which is a synthetic ester dielectric liquid designed to provide an alternative to flammable mineral oil. Since the advent of MIDEL 7131 in the 1970s, this liquid (with very low conductivity and higher permittivity $\varepsilon \approx 3.1$, as well as superior oxygen and moisture stability) has gained an excellent reputation as an effective dielectric, not only in industrial high-voltage transformers, but also in pulsed power systems as well $[14,34]$. Besides the possibility of decreasing the PFL length by $17 \%$ at a specified pulse width, MIDEL 7131 can provide a gain in the PFL outer dimension due to its high breakdown strength $E_{\mathrm{br}}$ and the stability of its electric parameters during long-term operation [35]. For example, if $E / E_{\mathrm{br}}$ is kept stable at $\approx 0.37$ or a lower level, a lifetime of up to $10^{8}$ cycles or more can be expected [14]. The breakdown strength $(\mathrm{MV} / \mathrm{cm})$ can be expressed by an empirical equation such as that of $E_{\mathrm{br}}$ for mineral oil, with large-surface electrodes in a weakly inhomogeneous field. The empirical equation has the form [30]:

$$
E_{\mathrm{br}}=A /\left(t_{\mathrm{eff}}^{0.33} \cdot S^{0.08}\right)
$$

where $A$ is a constant equal to $\approx 0.6$ at normal pressure and $\approx 1.2$ at $1.2 \mathrm{MPa}[25,31], t_{\mathrm{eff}}$ is the effective time $(\mu \mathrm{s})$ for which the field strength is higher than $0.63 E_{\mathrm{b} r}\left(t_{\mathrm{eff}} \approx 0.25 t_{\mathrm{ch}}\right)$, and 
$S\left(\mathrm{~cm}^{2}\right)$ is the electrode area. In experiments using MIDEL 7131 [14], $E_{\mathrm{br}} \approx 0.38 \mathrm{MV} / \mathrm{cm}$ and $E \approx 0.14 \mathrm{MV} / \mathrm{cm}$ were provided.

For optimum PFL-load matching, it is required that the maximum energy at arbitrary $R_{L} \neq \rho$ be the energy transferred to the first (main) pulse. With constant $r_{2}, E$, and $R_{\mathrm{L}}$, the optimum value $\Lambda_{\text {opt }}$ is determined by Formula (2) in [1], in the form:

$$
\Lambda_{\mathrm{opt}}=(\alpha / 2)\left\{[1+(4 / \alpha)]^{1 / 2}-1\right\} \text { with } \alpha=R_{\mathrm{L}} \varepsilon^{1 / 2} / 60
$$

Then, for $R_{\mathrm{L}} \approx 100 \Omega$, the optimum PFL impedance is $\rho_{\text {opt }} \approx 30.6 \Omega$ (by Equation (4), $\eta \approx 0.718)$ at $\varepsilon=2.25$ and $\rho_{\text {opt }} \approx 26.9 \Omega(\eta \approx 0.668)$ at $\varepsilon=3.1$. The matching efficiency can be increased by separating high-voltage pulses into several channels, because the number of channels determines (equals) the factor of decrease in the effective load resistance. For example, such an increase in matching efficiency is provided by pulse separation into two [11,12] and four channels with a pulse sharpener in each of them [36,37]. With phasecontrolled parallel channels in shock-excited, nanosecond microwave oscillators [9], one can increase the total power and provide microwave beam control similar to that in active antenna arrays [37].

Another method of increasing the matching efficiency ( $\eta$ up to unit) and the voltage pulse width is to insert additional uncharged lines into a PFL, such that its effective wave impedance will increase by an integer number $k=2,3, \ldots$.

\section{Results}

\subsection{Theoretical Grounds for Extended FLs}

\subsubsection{Double-Width Forming Line}

Figure 3 shows a double-width forming line (DWFL) that comprises two seriesconnected lines $L_{0}, L_{1}$ that are equal in electrical length $T$ and wave impedance $\rho$ [23]. At the time of switch operation, line $L_{0}$ is charged to $U_{0}$, and line $L_{1}$ is uncharged.

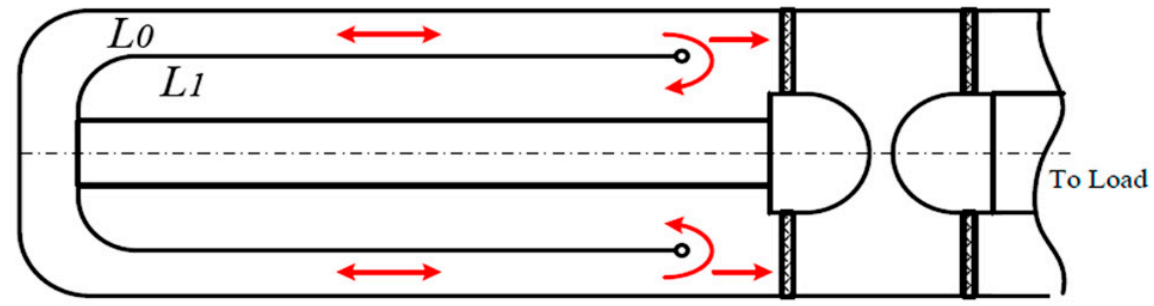

Figure 3. Double width forming line.

Obviously, when discharged, the DWFL equivalent impedance is $2 \rho$, and hence the load resistance is taken to be $R_{\mathrm{L}}=2 \rho$. A voltage pulse of amplitude $U_{0} / 2$ and duration $4 T$ is provided at the load by the DWFL discharge circuit (Figure 4).

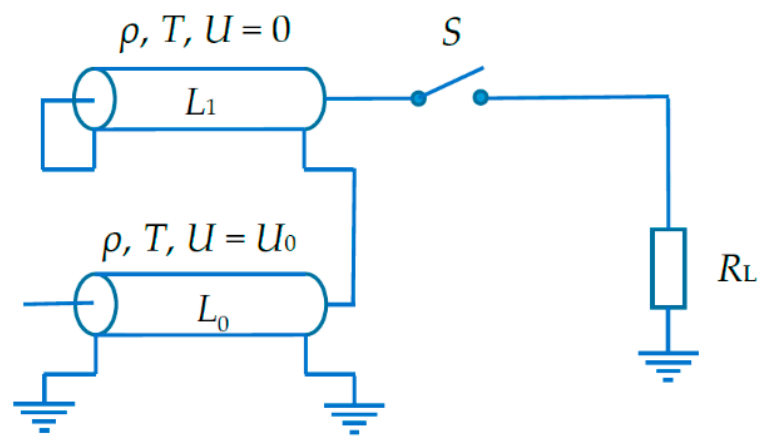

Figure 4. DWFL discharge circuit.

Immediately after operation of the switch $(S)$, the circuit begins drawing a current $I=U_{0} /\left(R_{\mathrm{L}}+2 \rho\right)=U_{0} / 4 \rho$, which provides a voltage pulse amplitude $U_{\mathrm{L}}=I \cdot R_{\mathrm{L}}=U_{0} / 2$ at 
the load. Simultaneously, a voltage wave of amplitude $U_{\mathrm{b}}=I \cdot \rho=U_{0} / 4$ propagates from right to left in the lines $L_{0}, L_{1}$. By the time $t=T$, the wave front in $L_{0}$ reaches its open end at $3 U_{0} / 4$, and the wave front in $L_{1}$ reaches the short-circuited end at $-U_{0} / 4$ and goes back with reversed polarity. By the time $t=2 T$, both waves return to the load such that the wave amplitude becomes $U_{0} / 2$ in $L_{0}$ and zero in $L_{1}$. For estimating the wave amplitudes in the two lines at the next time points, we can introduce their transmission and reflection coefficients $k^{+}$and $k^{-}$(to and from the load, respectively). For $L_{0}$ and $L_{1}$, these coefficients are equal and given by:

$$
\begin{gathered}
k^{+}=2\left(R_{\mathrm{L}}+\rho\right) /\left[\left(R_{\mathrm{L}}+\rho\right)+\rho\right]=3 / 2 ; \text { and } \\
\left.\left.k^{-}=\left[R_{\mathrm{L}}+\rho\right)-\rho\right] /\left[R_{\mathrm{L}}+\rho\right)+\rho\right]=1 / 2
\end{gathered}
$$

Thus, considering that the $L_{0}$ wave at $2 T<t<3 T$ is divided into series-connected $R_{\mathrm{L}}$ and $\rho$, we have $(3 / 2)(2 / 3)(1 / 2) U_{0}=U_{0} / 2$ at the load, a uniform decrease in the $L_{0}$ wave amplitude from $U_{0} / 2$ at $2 T$ to $U_{0} / 4$ at $3 T$, and $U_{0} / 8+U_{0} / 8=U_{0} / 4$ throughout the $L_{1}$ line.

On the interval $3 T<t<4 T$, the $L_{0}$ and $L_{1}$ waves propagating to the load occupy increasingly less space, providing $U_{0} / 2$ at the load. Thus, the PFL comprising a shortcircuited segment with a wave impedance $\rho$ and electrical length $T$ can double the load pulse width and halve the load power.

\subsubsection{Triple-Width Forming Line}

Figures 5 and 6 show a triple-width forming line (TWFL) and its discharge circuit, respectively.

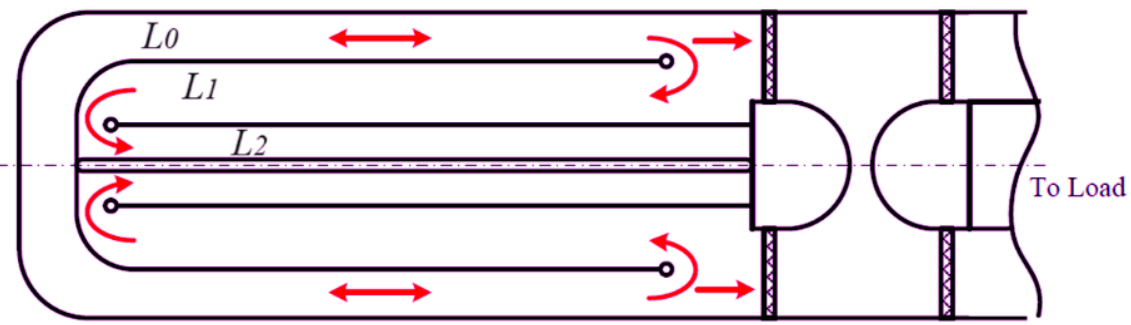

Figure 5. Simplified TWFL schematic.

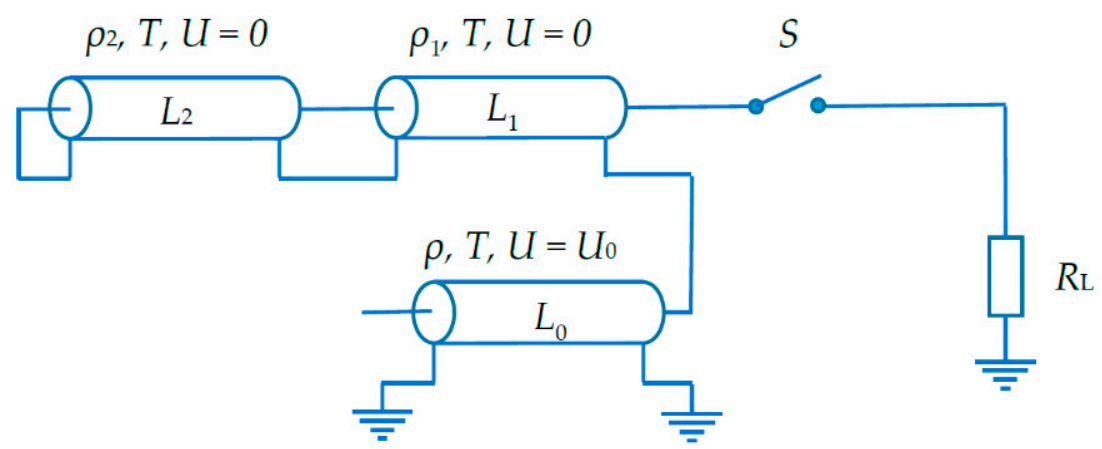

Figure 6. TWFL discharge circuit.

At the initial time point, $L_{0}$ is charged to $U_{0}$ while $L_{1}$ and $L_{2}$ are uncharged. The electrical lengths of $L_{0}, L_{1}$, and $L_{2}$ are equal to $T$; the wave impedance of $L_{0}$ is $\rho$. The coefficient of pulse extension $k=3$ determines the load resistance $R_{\mathrm{L}}=3 \rho$. Considering that the load wave amplitude at $0<t<T$ is $U_{0} / 2$ and that the current is $I=U_{0} /\left(R_{\mathrm{L}}+\rho+\rho_{1}\right)$, the impedance of $L_{1}$ can be estimated as $\rho_{1}=2 \rho$ and the current as $I=U_{0} / 6 \rho$. The backward wave amplitudes in $L_{0}$ and $L_{1}$ are $-I \rho=-U_{0} / 6$ and $-I \rho_{1}=-U_{0} / 3$, respectively. Thus, by the point $t=T$, the wave amplitude is $-U_{0} / 3$ in $L_{1}$, zero in $L_{2}$, and $5 / 6 U_{0}$ in $L_{0}$. The backward voltage wave in $L_{0}$ reaches the plane with a circuit break and goes back with the same polarity. 
The reflected wave amplitude $U$ can be estimated in $L_{2}$ for $T<t<2 T$ by reasoning that the resulting amplitude at the load should be $U_{0} / 2: U^{-}-U_{0} / 3+2 / 3 U_{0}=U_{0} / 2$. We obtain $U^{-}=U_{0} / 6$. Thus, the voltage across $L_{1}$ is $U_{L 1}=-U_{0} / 3+U^{-}=-U_{0} / 6$. However, this is possible at a definite reflection coefficient $k^{-}=\left(\rho_{2}-\rho_{1}\right) /\left(\rho_{2}+\rho_{1}\right)$ whose value from $-\mathrm{k}-U_{0} / 3=U_{0} / 6$ is $k^{-}=-1 / 2$. Hence, $\rho_{2}=\rho_{1} / 3=2 \rho / 3$. By the time point $t=2 T$, the wave amplitude in $L_{1}$ and in $L_{2}$ is $-U_{0} / 6$, and in $L_{0}$, it is $2 / 3 U_{0}$. The next time points are only important for estimating the wave amplitudes in each line. For such estimation, the transmission and reflection coefficients at two boundaries are used. Omitting simple calculations, we obtain that by the point $t=3 T$, the wave amplitudes in $L_{1}$ and $L_{2}$ become equal to zero, and the wave amplitude in $L_{0}$ is $1 / 2 U_{0}$. Upon completion of the processes at $3 T<t<4 T$, the wave amplitudes in $L_{1}$ and $L_{2}$ are $U_{0} / 6$, and the wave amplitude in $L_{0}$ is $1 / 3 U_{0}$.

By the time point $t=5 T$, the wave amplitude in $L_{1}$ and in $L_{2}$ increases to $U_{0} / 3$, and in $L_{0}$, it decreases to $1 / 6 U_{0}$. Finally, at $t=6 T$, the voltage amplitudes in all lines are equal to zero. Table 1 presents the voltage amplitudes in each line at different time points.

Table 1. Voltage amplitudes in TWFL.

\begin{tabular}{cccc}
\hline $\boldsymbol{t}$ & $\boldsymbol{U}_{\mathbf{L} \mathbf{0}} / \boldsymbol{U}_{\mathbf{0}}$ & $\boldsymbol{U}_{\mathbf{L} \mathbf{1}} / \boldsymbol{U}_{\mathbf{0}}$ & $\boldsymbol{U}_{\mathrm{L} \mathbf{2}} / \boldsymbol{U}_{\mathbf{0}}$ \\
\hline 0 & 1 & 0 & 0 \\
$T$ & $5 / 6$ & $-1 / 3$ & 0 \\
$2 T$ & $2 / 3$ & $-1 / 6$ & $-1 / 6$ \\
$3 T$ & $1 / 2$ & 0 & 0 \\
$4 T$ & $1 / 3$ & $1 / 6$ & $1 / 6$ \\
$5 T$ & $1 / 6$ & $1 / 3$ & $1 / 3$ \\
$6 T$ & 0 & 0 & 0 \\
\hline
\end{tabular}

Thus, the PFL configuration with two nested lines provides a rectangular voltage pulse of amplitude $U_{\mathrm{L}}=U_{0} / 2$ at a matched load and width $t=6 T$. The effective internal impedance of the TWFL is $3 \rho$.

\subsubsection{Forming Line with k-Fold Extension}

The possibility of two- and three-fold pulse lengthening and the similarity of DWFL and TWFL discharge processes suggest that a configuration with a higher integer factor of pulse extension $k$ can be designed in which the number of additional lines is $k-1$. In this case, the matched load resistance should increase to $R_{\mathrm{L}}=k \rho$. The impedance of $L_{1}$ (additional line) is expressed as:

$$
\rho_{1}=R_{\mathrm{L}}-\rho=(k-1) \rho
$$

The discharge current after the switch operation is $I=U_{0} /\left(R_{\mathrm{L}}+\rho_{1}+\rho\right)=U_{0} /(2 k \rho)$. At the load, $U_{\mathrm{L}}=I \cdot R_{\mathrm{L}}=U_{0} / 2$. The backward wave amplitude in $L_{0}$ is $-I \rho=-U_{0} / 2 k$, and in $L_{1}$, it is $-I \rho_{1}=-U_{0}(k-1) / 2 k$. The impedance of $L_{2}$ is chosen for $T<t<2 T$ from the condition that the load voltage is $U_{0} / 2$. Similarly, we estimate the impedance of $L_{3}$ for $2 T<t<3 T$. Omitting intermediate calculations, we obtain:

$$
\rho_{2}=\rho_{1}(k-2) / k
$$

The impedances of nested lines can be written in the form of a recurrence formula for $k \geq 3$ as a generalization of the TWFL case with $k=3$ :

$$
\rho_{\mathrm{n}}=\rho_{\mathrm{n}-1}\{(k-n) /(k+2-n)\}
$$

where $n=2, \ldots,(k-1), k \geq 3$.

It is noteworthy that the time behavior of voltage amplitudes in each line obeys rather simple laws that can easily be written, even from the data in Table 1 . In particular, the voltage across the main line $\left(L_{0}\right)$ decreases by $1 / 2 k$ from its initial value $U_{0}$ on each interval 
$T$, and the voltage amplitude across the nested lines, being kept in the range $( \pm 1 / k) \cdot U_{0}$, increases by $1 / 2 k$ from its value at the previous stage.

\subsection{Simulation Results for TWFL}

Our computations and experiments were performed for a dielectric with a relative permittivity $\varepsilon=2.25$, which corresponded to transformer oil. The forming line was a TWFL with a length $L_{\mathrm{FL}}=120 \mathrm{~cm}$ and main-line conductor radii $r_{2}=15.6 \mathrm{~cm}$, and $r_{1}=9.8 \mathrm{~cm}$ (Figure 7).
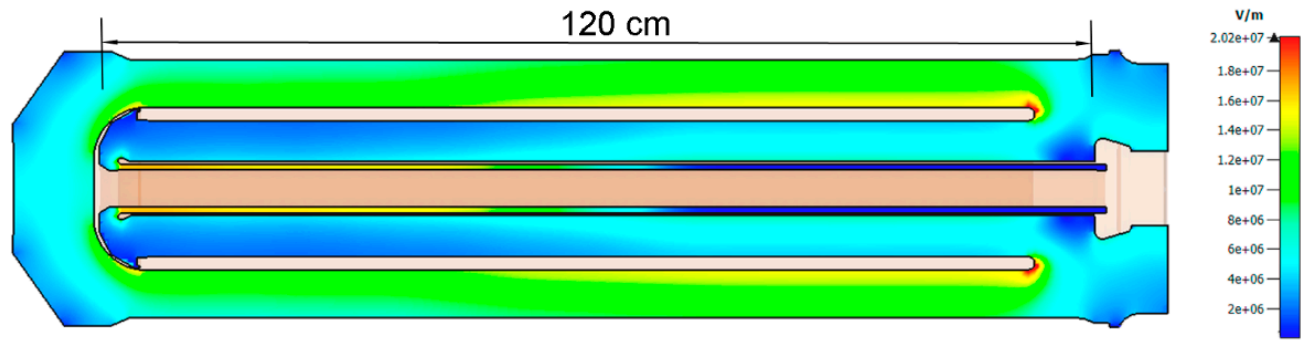

Figure 7. Simulation results on field distribution in real TWFL at discharge point of $10 \mathrm{~ns}$.

The discharge processes were simulated in the environment of CST Studio Suite software with schematic and 3D programs. For the TWFL parameters used, the impedance of the main line was $\rho=18.6 \Omega$. The switch was perfect, with a series-connected resistance equal to $18.6 \cdot 3=55.8 \Omega$. According to Equations (7) and (8), the wave impedance of two nested lines should be $\rho_{1}=37.2 \Omega$ and $\rho_{2}=12.4 \Omega$, respectively. In the simulation, the charging voltage was $U_{0}=794 \mathrm{kV}$, for which Equation (2) gave $E=0.175 \mathrm{MV} / \mathrm{cm}$ in the main line before discharging. According to electrostatic computations, the local field at the right electrode end, rounded to a radius of $0.8 \mathrm{~cm}$, was no greater than $0.24 \mathrm{MV} / \mathrm{cm}$.

In the experimental configuration, the gap of the inner coaxial deviated from its theoretical value such that $\rho_{2}$ was $10.8 \Omega\left(13 \%\right.$ lower than the needed value for $\left.\rho_{2}\right)$. Therefore, the same computations were performed for two configurations: theoretical (ideal) and experimental (real).

As can be seen from Figure 8, the voltage pulse in the real TWFL revealed two steps, deviating by no more than $\pm 5 \%$ from the amplitude of the first third.

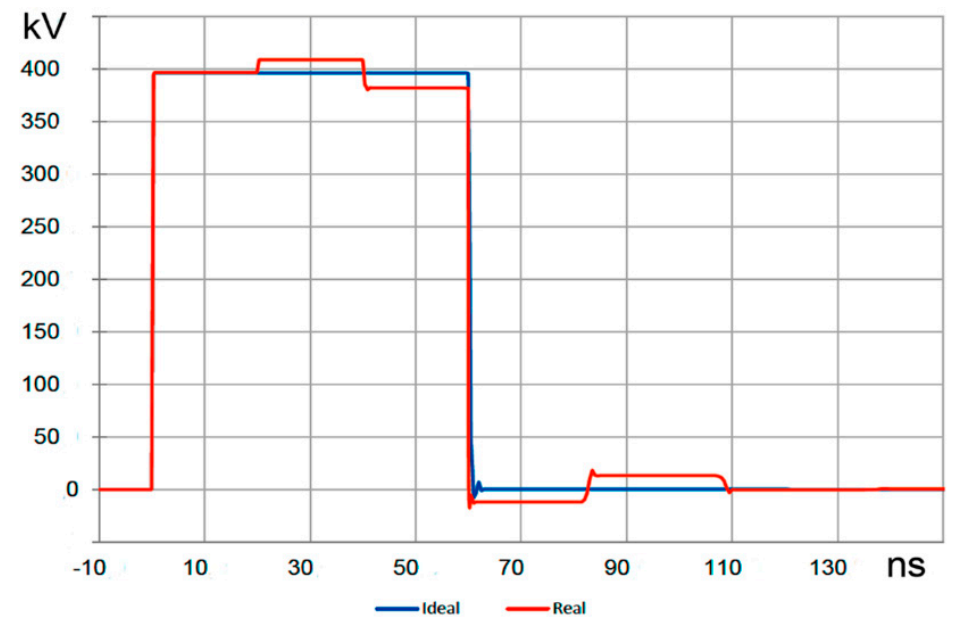

Figure 8. Computed voltage pulse shapes in ideal TWFL with $\rho_{2}=12.4 \Omega$ (blue) and real TWFL with $\rho_{2}=10.8 \Omega$ (red).

The simulation showed that the field strength at the electrodes of all coaxial lines and in all discharge stages corresponded to the time variation of their potential, exactly as given in Table 1. In particular, a significant issue can be the electrical strength of the inner gap of 
the line with the lowest impedance. Figure 9 demonstrates the field distribution at the time point $25 \mathrm{~ns}$, which lay in the range $4 T<t<5 T$ ( $T=6 \mathrm{~ns}$, see Table 1 ) and corresponded to the maximum potential difference in this part of the structure.
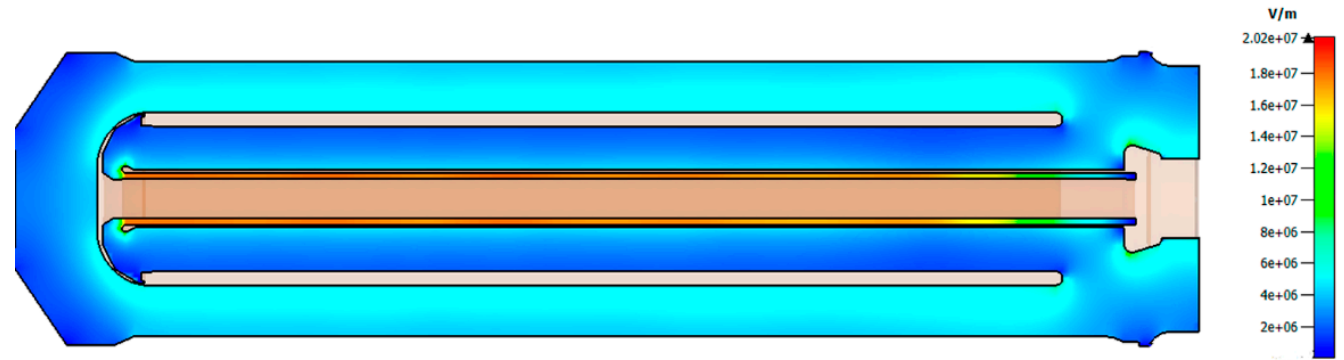

Figure 9. Field distribution in TWFL at discharge point $25 \mathrm{~ns}$.

It is seen that the field in this TWFL part and at the rounded electrode end was not higher than $0.23 \mathrm{MV} / \mathrm{cm}$. By considering the time of insulation exposure to the field and from the electrode surface areas (see Equation (5)), it could be concluded that the breakdown strength of the composite versions was approximately the same at the single PFL. What was intriguing is that this conclusion held for all inner electrode surfaces, despite the decrease in the transverse dimensions.

\subsection{Laboratory Model of SINUS-Type Driver Based on TWFL}

Figure 10 shows a laboratory model of a TWFL, which is a modification of the SINUS330 setup based on a PFL of outer diameter $33 \mathrm{~cm}$.

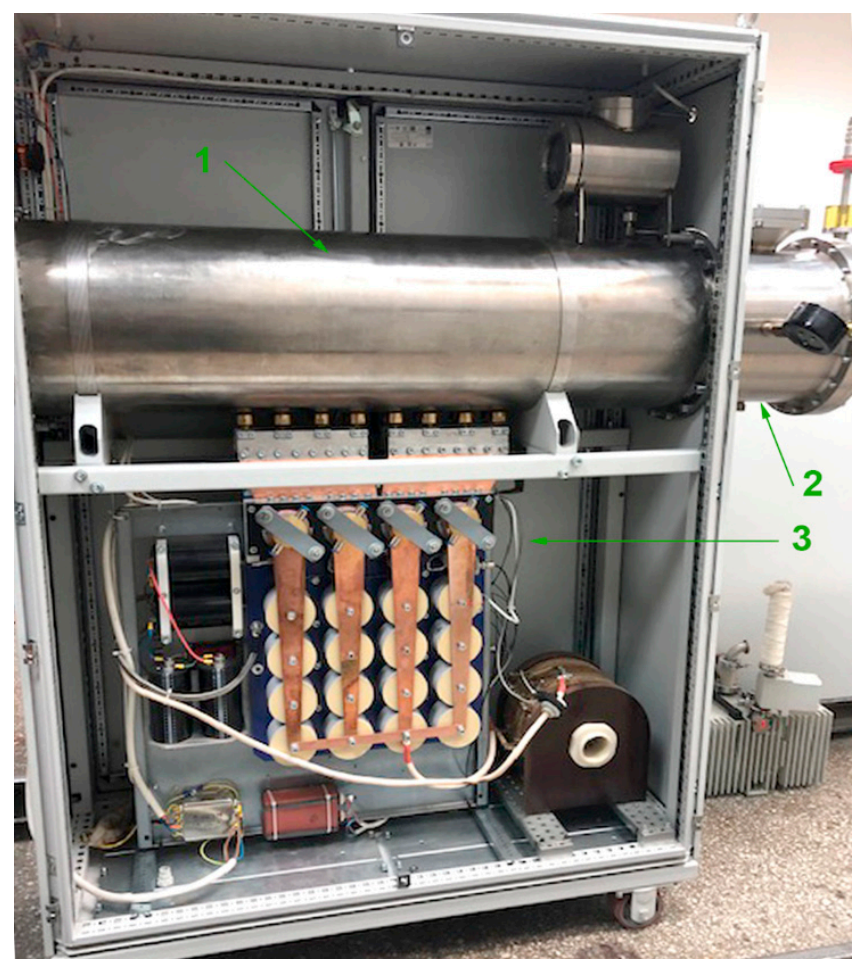

Figure 10. TWFL-based SINUS driver for low pulse repetition rates: 1-Tesla transformer with TWFL; 2-gas-gap switch chamber; 3-primary transformer circuit with thyristors, capacitors, and other elements.

Originally, the SINUS-330 setup (a single FL with $\rho_{0} \approx 19 \Omega$ and $L_{\mathrm{FL}} \approx 120 \mathrm{~cm}$ ) was designed for experiments with Cherenkov-type microwave oscillators with a millimeter wavelength range. A typical waveform of the cathode voltage is shown in Figure 11. 


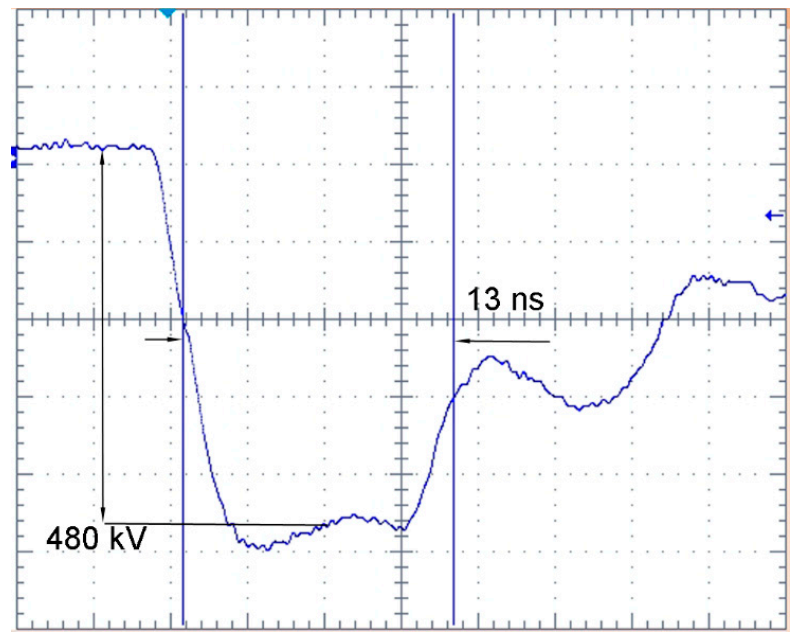

Figure 11. Voltage waveform at the resistive load for single PFL charged to $-600 \mathrm{kV}$ (time base $4 \mathrm{~ns} / \mathrm{div}$ ).

Such research does not require high pulse repetition rates due to the use of impulse magnetic systems capable of providing a few pulses per minute for beam formation and transport. Compared to high repetition rates, the total number of pulses producible within a limited time is many (e.g., four) orders of magnitude lower. Therefore, the ratio $E / E_{\mathrm{b} r}$ may be increased; e.g., through increasing the charging voltage $U_{0}$ by $30-40 \%[1,26]$. In addition, no forced gas circulation is needed for the gas-gap switch (2) and the primary transformer circuit (3) is simplified as well.

With a fixed gap in the gas-gap switch, the charging voltage $U_{0}$ could be varied from 580 to $670 \mathrm{kV}$, by varying the gas (nitrogen) pressure in the chamber.

With the original PFL (Figure 11), the voltage pulse duration across the load was $\approx 13 \mathrm{~ns}$ at a level of $0.5 U_{\max }$. The voltage rise and fall times at $0.1-0.9 U_{\max }$ were about $4 \mathrm{~ns}$. Thus, the pulse had a relatively short quasi-flat top of $\approx 10 \mathrm{~ns}$. The load comprised high-voltage resistors with a total resistance of $80 \Omega$ in the operating voltage range.

With its TWFL version at the same stored energy (Figure 12), the flat top of the pulse increased more than three times, and the voltage rise time decreased to about $3 \mathrm{~ns}$. The voltage waveform was quite close to that observed in the simulation. The overshot, after a rise time of about $3 \mathrm{~ns}$, was due to the presence of a low-impedance piercing of the initial part of the transmission line, which was not used in the simulation. The diode voltage amplitude $U_{\mathrm{L}} \approx 380 \mathrm{kV}$ corresponded to the charging voltage $\left(U_{0} \approx 650 \mathrm{kV}\right)$ and the value of the load $\left(R_{\mathrm{L}} \approx 80 \Omega\right): U_{\mathrm{L}}=U_{0} R_{\mathrm{L}} /\left(R_{\mathrm{L}}+\rho_{\text {eff }}\right)$, where $\rho_{\text {eff }} \approx 56 \Omega$. Equation (4) calculates the matching efficiency of the high-voltage driver and the load $96.9 \%$.

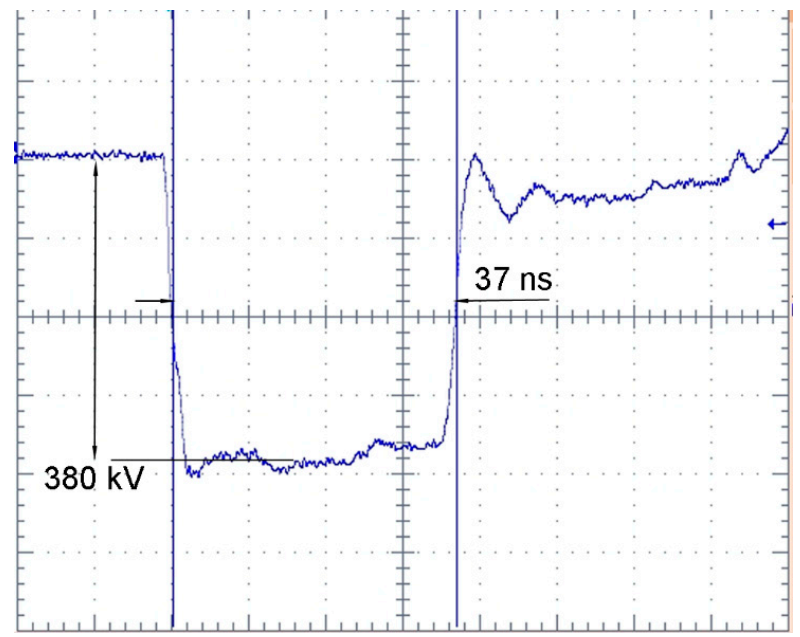

Figure 12. Voltage waveform across resistive load for TWFL charged to $-650 \mathrm{kV}$ (time base $10 \mathrm{~ns} /$ div). 


\section{Discussion}

At equal stored energies, TWFLs $(k=3)$ have the same advantages as DWFLs over conventional PFLs [23] when high-resistance loads are used. The pulse extension allows one to decrease the driver dimensions at a specified load pulse duration, to increase the efficiency of matching the driver and the load, and to shorten the pulse rise time. It was supposed that the decrease in the voltage rise time was associated with the spark-channel dynamics, depending on the internal generator impedance [25].

Obviously, passing to longer high-voltage pulses needs a careful consideration of the reliability of the transmission-line insulation, including the solid-state vacuum insulator.

The proposed method of 4 -fold voltage pulse extension $(k=4)$ has the prospect of increasing the duration of microwave pulses in the millimeter wavelength range, at moderate and low accelerating voltages $(\approx 320 \mathrm{kV}$ and less) when the e-beam diode impedance should measure $\approx 120 \Omega$ or higher $[12,17]$.

\section{Conclusions}

The proposed method allowed a many-fold extension of a high-voltage pulse by nesting additional lines into the free volume of the inner electrode of an ordinary PFL. For the first time, the general requirements for such lines have been formulated. The method has been given theoretical grounds. Its efficiency has been proven in a practice earlier for an X-band repetitively pulsed HPM relativistic backward wave oscillator [14] that was driven by a double-width pulse-forming line: for a fixed microwave pulse duration ( $20 \mathrm{~ns})$, the total length of the source was decreased by approximately two times. The expectations of high efficiency of driver-load matching were confirmed in the described experiment with a triple-width forming line.

Author Contributions: Conceptualization, V.V.R. and A.S.S.; Data curation, V.V.R., A.S.S., P.V.V. and R.V.T.; Formal analysis, V.V.R., A.S.S. and R.V.T.; Investigation, R.V.T.; Methodology, V.V.R. and A.S.S.; Software, A.S.S. and R.V.T.; Validation, A.S.S., P.V.V. and R.V.T.; Visualization, A.S.S.; Writing — original draft, V.V.R. and A.S.S.; Writing—review and editing, V.V.R. All authors have read and agreed to the published version of the manuscript.

Funding: This research was supported by the Ministry of Science and Higher Education of the Russian Federation (No FWRM-2021-0002).

Conflicts of Interest: The authors declare no conflict of interest.

\section{References}

1. Mesyats, G.A.; Korovin, S.D.; Gunin, A.V.; Gubanov, V.P.; Stepchenko, A.S.; Grishin, D.M.; Landl, V.F.; Alekseenko, P.I. Repetitively pulsed high-current accelerators with transformer charging of forming lines. Laser Part. Beams 2003, 21, 197-209. [CrossRef]

2. Zhang, H.; Shu, T.; Liu, S.; Zhang, Z.; Song, L.; Zhang, H. A Compact Modular 5 GW Pulse PFN-Marx Generator for Driving HPM Source. Electronics 2021, 10, 545. [CrossRef]

3. Lassale, F.; Morell, A.; Loyen, A.; Chanconie, T.; Roques, B.; Toury, M.; Vezinet, R. Development and test of a 400-kV PFN Marx with compactness and rise time optimization. IEEE Trans. Plasma Sci. 2018, 46, 3313-3319. [CrossRef]

4. Krasik, Y.E.; Dunaevsky, A.; Krokhmal, A.; Felsteiner, J.; Gunin, A.V.; Pegel, I.V.; Korovin, S.D. Emission properties of different cathodes at $\mathrm{E} \leq 10^{5} \mathrm{~V} / \mathrm{cm}$. J. Appl. Phys. 2001, 89, 2379-2399. [CrossRef]

5. Afanas'ev, K.V.; Bykov, N.M.; Gubanov, V.P.; El'chaninov, A.A.; Klimov, A.I.; Korovin, S.D.; Rostov, V.V.; Stepchenko, A.S. A high-power periodic nanosecond pulse source of coherent 8-cm electromagnetic radiation. Tech. Phys. Lett. 2006, 32, 925-927. [CrossRef]

6. Shafir, G.; Kreif, M.; Gleizer, J.Z.; Krasik, Y.E.; Gunin, A.V.; Kutenkov, O.P.; Pegel, I.V.; Rostov, V.V. Experimental research of different plasma cathodes for generation of high-current electron beams. J. Appl. Phys. 2015, 118, 193302. [CrossRef]

7. Belomyttsev, S.Y.; Rostov, V.V.; Romanchenko, I.V.; Shunailov, S.A.; Kolomiets, M.D.; Mesyats, G.A.; Sharypov, K.A.; Shpak, V.G.; Ulmaskulov, M.R.; Yalandin, M.I. Magnetically insulated coaxial vacuum diode with partial space-charge-limited explosive emission from edge cathode. J. Appl. Phys. 2016, 119, 023304. [CrossRef]

8. Belomyttsev, S.Y.; Korovin, S.D.; Pegel, I.V. Effect of the expanding explosive-emission centers plasma on the impedance of a high-current diode. IEEE Trans. Plasma Sci. 1999, 27, 1572-1577. [CrossRef] 
9. Rostov, V.V.; El'chaninov, A.A.; Klimov, A.I.; Konev, V.Y.; Romanchenko, I.V.; Sharypov, K.A.; Shunailov, S.A.; Ul'maskulov, M.R.; Yalandin, M.I. Phase control in parallel channels of shock-excited microwave nanosecond oscillators. IEEE Trans. Plasma Sci. 2013, 41, 2735-2741. [CrossRef]

10. Yalandin, M.I.; Reutova, A.G.; Ulmaskulov, M.R.; Sharypov, K.A.; Shpak, V.G.; Shunailov, S.A.; Klimov, A.I.; Rostov, V.V.; Mesyats, G.A. Picosecond stability of injection of parallel high-current electron beams. Tech. Phys. Lett. 2009, 35, 804-807. [CrossRef]

11. Rostov, V.V.; Elchaninov, A.A.; Romanchenko, I.V.; Yalandin, M.I. A coherent two-channel source of Cherenkov superradiance pulses. Appl. Phys. Lett. 2012, 100, 224102. [CrossRef]

12. Rostov, V.V.; Elchaninov, A.A.; Romanchenko, I.V.; Shunailov, S.A.; Ulmaskulov, M.R.; Sharypov, K.A.; Shpak, V.G.; Rukin, S.N.; Yalandin, M.I. Two-channel generator of the 8-mm wavelength range for radiation with subgigawatt power level pulses. Radiophys. Quantum Electron. 2014, 56, 475-491. [CrossRef]

13. Tot'meninov, E.M.; Vykhodsev, P.V.; Gunin, A.V.; Klimov, A.I.; Rostov, V.V. Increase in the energy efficiency of a pulsed-periodic relativistic backward wave oscillator with a modulating resonant reflector. Tech. Phys. 2014, 59, 428-433. [CrossRef]

14. Rostov, V.V.; Tsygankov, R.V.; Vykhodsev, P.V.; Konev, V.Y.; Stepchenko, A.S. Stable operation of a repetitively pulsed X-band relativistic backward wave oscillator. IEEE Electron Device Lett. 2021, 42, 935-938. [CrossRef]

15. Rostov, V.V.; Gunin, A.V.; Tsygankov, R.V.; Romanchenko, I.V.; Yalandin, M.I. Two-wave Cherenkov oscillator with moderately oversized slow-wave structure. IEEE Trans. Plasma Sci. 2017, 46, 33-42. [CrossRef]

16. Sun, J.; Chen, C. Research on 3-GW repetitively operating relativistic backward wave oscillator. IEEE Trans. Plasma Sci. 2020, 48, 3535-3543. [CrossRef]

17. Bratman, V.L.; Denisov, G.G.; Kolchugin, B.D.; Korovin, S.D.; Polevin, S.D.; Rostov, V.V. Powerful millimeter-wave generators based on the stimulated Cerenkov radiation of relativistic electron beams. Int. J. Infrared Millim. Waves. 1984, 5, 1311-1332. [CrossRef]

18. Denisov, G.G.; Smorgonsky, A.V.; Gubanov, V.P.; Korovin, S.D.; Rostov, V.V.; Yalandin, M.I. Powerful electromagnetic millimeterwave oscillations produced by stimulated scattering of microwave radiation by relativistic electron beams. Int. J. Infrared Millim. Waves. 1984, 5, 1389-1403. [CrossRef]

19. Korovin, S.D.; Rostov, V.V.; Smorgonsky, A.V. Pulse-periodic relativistic carsinotron (in Russian). Izv. Vyssh. Uchebn. Zaved. Radiofiz. 1986, 29, 1278-1280.

20. Gubanov, V.P.; Korovin, S.D.; Pegel, I.V.; Roitman, A.M.; Rostov, V.V.; Stepchenko, A.S. Compact 1000 pps high-voltage nanosecond pulse generator. IEEE Trans. Plasma Sci. 1997, 25, 258-265. [CrossRef]

21. Gunin, A.V.; Landl', V.F.; Korovin, S.D.; Mesyats, G.A.; Rostov, V.V. Long-lived explosive-emission cathode for high-power microwave generators. Tech. Phys. Lett. 1999, 25, 922-926. [CrossRef]

22. Zhang, J.; Jin, Z.-X.; Yang, J.-H.; Zhong, H.-H.; Shu, T.; Zhang, J.-D.; Qian, B.-L.; Yuan, C.-W.; Li, Z.-Q.; Fan, Y.-W.; et al. Recent advance in long-pulse HPM sources with repetitive operation in S.-, C.-, and X-bands. IEEE Trans. Plasma Sci. 2011, 39, 1438-1445. [CrossRef]

23. Liu, S.; Su, J.-C.; Zhang, X.; Pan, Y.-F.; Fan, H.-Y.; Fan, X.-L. A Tesla-type long-pulse generator with wide flat-top width based on double-width pulse-forming line. Laser Part. Beams 2018, 36, 115-120. [CrossRef]

24. Eltchaninov, A.S.; Zagulov, F.Y.; Korovin, S.D.; Mesyats, G.A. Electron beam accelerator with high pulse recurrence frequency In Proceedings of the 3rd International Topical Conference on High Power Electron and Ion Beams, Novosibirsk, Russia, 3-6 July 1979; pp. 191-197.

25. Mesyats, G.A. Pulsed Power, 1st ed.; Springer: Boston, MA, USA, 2005. [CrossRef]

26. Korovin, S.D.; Rostov, V.V.; Polevin, S.D.; Pegel, I.V.; Schamiloglu, E.; Fuks, M.I.; Barker, R.J. Pulsed power-driven high-power microwave sources. Proc. IEEE 2004, 92, 1082-1095. [CrossRef]

27. Mesyats, G.A.; Korovin, S.D.; Rostov, V.V.; Shpak, V.G.; Yalandin, M.I. The RADAN series of compact pulsed power generators and their applications. Proc. IEEE 2004, 92, 1166-1179. [CrossRef]

28. Zhang, Z.; Zhang, J.; Qian, B.; Liu, C.; Xun, T.; Zhang, H.; Liang, B. Compact rep-rate GW pulsed generator based on forming line with built-in high-coupling transformer. IEEE Trans. Plasma Sci. 2014, 42, 241-248. [CrossRef]

29. Liu, S.F.; Zhang, Z.C.; Wang, Y.W.; Zhang, J.D.; Xiao, L.A.; Liang, B.; Li, D.G. Low-impedance high-power pulsed generator based on forming line with built-in Tesla transformer. Rev. Sci. Instrum. 2021, 92, 084705. [CrossRef]

30. Martin, J.C. Nanosecond pulse techniques. Proc. IEEE 1992, 80, 934-945. [CrossRef]

31. Ushakov, V.Y. Behavior of Liquids in Strong Electric Fields. In Impulse Breakdown of Liquids; Power Systems; Springer: Berlin/Heidelberg, Germany, 2007; pp. 1-51. [CrossRef]

32. Korovin, S.D.; Rostov, V.V.; Tot'meninov, E.M. A relativistic backward wave oscillator with a modulating resonance reflector. Tech Phys. Lett. 2005, 31, 411-413. [CrossRef]

33. Tot'meninov, E.M.; Klimov, A.I.; Kurkan, I.K.; Polevin, S.D.; Rostov, V.V. Repetitively pulsed relativistic BWO with enhanced mechanical frequency tunability. IEEE Trans. Plasma Sci. 2008, 36, 2609-2612. [CrossRef]

34. Liu, S.; Su, J.; Fan, X. Note: A high-energy-density tesla-type pulse generator with novel insulating oil. Rev. Sci. Instrum. 2017, 88, 096101. [CrossRef] [PubMed]

35. Martin, D.; Wang, Z.D. Statistical analysis of the AC breakdown voltages of ester based transformer oils. IEEE Trans. Dielectr. Electr. Insul. 2008, 15, 1044-1050. [CrossRef] 
36. Ulmaskulov, M.R.; Pedos, M.S.; Rukin, S.N.; Sharypov, K.A.; Shpak, V.G.; Shunailov, S.A.; Yalandin, M.I.; Romanchenko, I.V.; Rostov, V.V. High repetition rate multi-channel source of high-power rf-modulated pulses. Rev. Sci. Instrum. 2015, 86, 074702. [CrossRef] [PubMed]

37. Romanchenko, I.V.; Ulmaskulov, M.R.; Sharypov, K.A.; Shunailov, S.A.; Shpak, V.G.; Yalandin, M.I.; Pedos, M.S.; Rukin, S.N.; Konev, V.Y.; Rostov, V.V. Four channel high power rf source with beam steering based on gyromagnetic nonlinear transmission lines. Rev. Sci. Instrum. 2017, 88, 054703. [CrossRef] 